\title{
DE COMO EL TRANSNACIONALISMO FACILITA LA PARTICIPACIÓN DE LOS INMIGRANTES MEXICANOS EN LA POLITICA ESTADOUNIDENSE
}

\author{
PATRICIA H. ESCAMILLA HAMM*
}

RESUMEN: Este artículo examina el impacto del transnacionalismo político en la participación de los inmigrantes mexicanos en la política estadounidense. Se sugiere que la participación de los inmigrantes en la política mexicana facilita su intervención en los Estados Unidos en lugar de entorpecerla, como alegan algunos críticos. Apoyándose en investigaciones sobre transnacionalismo político y en un modelo de participación política, este artículo sugiere que las actividades transnacionales (por ejemplo, el cabildeo o la membresía en organizaciones de migrantes) permiten desarrollar experiencia y habilidades cívico-políticas que pueden utilizarse en el contexto estadounidense. El estudio se centra en el caso de algunos mexicanos involucrados en la campaña de cabildeo para promover la aprobación del voto ausente (1998-2006) y muestra lo que han aprendido a través del ejercicio del transnacionalismo y las maneras en que lo han utilizado en su actividad política en Estados Unidos. Con esto se contribuye al estudio del tema desde una nueva perspectiva y también se corroboran investigaciones previas al constatar que aquellos que participan regularmente en los procesos y asuntos políticos mexicanos no sólo no ignoran la política estadounidense sino que se involucran en ella de manera sustancial.

PALABRAS CLAVE: Inmigrantes mexicanos, participación política, política estadounidense, hometown associations, política mexicana.

ABSTRACT: This study examines the impact of political transnationalism on Mexican immigrants' participation in U.S. politics. The main argument is that involvement in Mexican politics -transnationalism- does not impede engagement in the U.S. political system, as critics claim. On the contrary, it facilitates and complements political participation in the United States. Drawing from the literature on transnationalism and theories of political participation, this study proposes that transnational activities -like lobbying and membership in immigrant organizations- offer opportunities to develop civic-political skills and experience that immigrants may in turn use in the U.S. political arena. By exploring the case of Mexican immigrants and organizations involved in the lobbying campaign on behalf of absentee-voting laws in Mexico (1998-2006), it identifies the abilities and practice acquired by participants and the ways in which they have used them to influence U.S. processes and issues. This work contributes to the literature by shedding light on themes that have not been previously examined from this perspective and by lending support to previous findings -especially the evidence that political transnationalists are habitually not detached from U.S. politics and, in fact, are inclined to participate.

KEYwORDS: Mexican immigrants, political participation, U.S. politics, hometown associations, mexican politics.

\footnotetext{
* Profesora-Investigadora. El Colegio de la Frontera Norte, Tijuana, Dept. of Public Administration, (664) 631-6330 ext.3407, escahamm@colef.mx
} 

bios constitucionales en relación a los derechos de los expatriados. Dichos cambios, realizados en 1996, eliminaron obstáculos legales previos que impedían votar desde el extranjero y mantener la nacionalidad mexicana a pesar de haber adquirido otra ciudadanía. Es decir, abrieron las puertas a la doble ciudadanía y la participación política desde fuera. Pronto, tanto involucrados como observadores comenzaron a preguntarse qué efecto tendrían estos cambios en la participación inmigrante en la política estadounidense. Un analista se preguntó si el voto transnacional daría lugar a una era «de declive electoral latino» (DA, 2003). ${ }^{1}$ Otro si los inmigrantes perderían el interés en la política de su nuevo país (Elias, 1998). En resumen, żcuáles serían las consecuencias de estos cambios en la presencia política de la comunidad latina estadounidense y el proceso de asimilación política de los inmigrantes mexicanos?

Los partidarios del transnacionalismo (es decir, de la participación política en la patria de origen) sostienen que este proceso nutre y refina la participación política en otros territorios. Pero críticos como Renshon (2005) y Huntington (2004) arguyen que mantener vínculos con el país de origen impide la asimilación y distrae a los inmigrantes de la vida política estadounidense. Este artículo explora los efectos del transnacionalismo inmigrante y se centra en el papel que puede llegar a jugar en la participación política en territorio estadounidense. La investigación utiliza fuentes tanto secundarias como primarias, sobre todo observaciones personales y entrevistas con líderes inmigrantes mexicanos residentes en Estados Unidos, activistas, y participantes en la campaña de cabildeo a favor del voto ausente $(1998-2006) .^{2}$

Antes de continuar es importante aclarar algunos conceptos fundamentales. La "participación política» (transnacional o nacional) se define, de acuerdo con Brady et al. (1995), como la participación en actividades electorales y no electorales como el voto, la candidatura, la contribución a campañas electorales (por ejemplo, donaciones o promoción de candidatos), el cabildeo, la participación en marchas y mítines, el seguimiento y discusión de eventos políticos, la organización, la movilización de grupos de base, la participación activa en asuntos comunitarios, y el quehacer voluntario. Los términos «participación transnacional», «actividades transnacionales» $y$ «transnacionalismo» se refieren a las acciones tomadas por individuos u organizaciones de expatriados que buscan participar en los pro-

1 Utilizo iniciales como DA para referirme a fuentes que entrevisté en vivo, por teléfono o correo electrónico. Estas fuentes no se incluyen en la sección de obras citadas.

2 Yo estuve presente en encuentros privados y públicos, foros y otros eventos organizados en lugares como Chicago, Los Ángeles, Orange County (California) y la Ciudad de México entre 2003 y 2006 con el objeto de entrevistar a los transnacionalistas involucrados y observar la dinámica del transnacionalismo político. También tuve conversaciones en vivo, por teléfono y correo electrónico con varios de ellos y les hice preguntas detalladas sobre sus actividades políticas transnacionales y en Estados Unidos. Finalmente, me dediqué a monitorear regularmente sus conversaciones en foros electrónicos e investigué otro tipo de fuentes, incluyendo periódicos, documentos personales y comunicados de prensa. 
cesos políticos de su país de origen (para una discusión detallada de estos conceptos, véanse Portes et al.). Finalmente, el término «transnacionalista» se refiere a los individuos y organizaciones que practican el transnacionalismo.

Se sugiere que el transnacionalismo suele complementar y facilitar la participación política en Estados Unidos en lugar de obstaculizarla. La evidencia del caso mexicano sugiere que la participación en asuntos pertinentes a la nación de origen desarrolla habilidades que fomentan la participación potencial en el sistema político estadounidense, incrementando así las posibilidades de participación real. Este estudio presenta nuevos datos sobre los recursos y experiencia cívico-políticos adquiridos por los inmigrantes, así como las maneras en que los han utilizado en el contexto estadounidense. Se sostiene la hipótesis de que los recursos adquiridos en un contexto particular pueden transferirse a otro (de doméstico a transnacional, de político a no político). Esto corrobora estudios previos acerca del impacto positivo del transnacionalismo, incluso aquellos que sugieren que, en lugar de distanciarse de la política estadounidense, los transnacionalistas probablemente habrán de participar en ésta.

\section{¿QUÉ SABEMOS ACERCA DEL TRANSNACIONALISMO POLÍTICO?}

El saber popular estadounidense sugiere que la única manera de lograr la asimilación política es cortando todo tipo de vínculos políticos con el país de origen. La doble nacionalidad, una forma cada vez más común de transnacionalismo político, se ha convertido en el blanco de críticas en ciertos círculos. Algunos de los participantes en un taller llevado a cabo por la Universidad de Duke en 1997 la caracterizaron como una distracción:

Si el afecto y compromiso de una persona se encuentran divididos, como bien puede ocurrir en el caso de la doble nacionalidad, éstos se verán disminuidos. Los dobles ciudadanos dedicarán menos tiempo a la participación cívica en la vida pública estadounidense ya que su lealtad involucrará a otro país (aunque sea de manera secundaria). (Pickus, 1997, 24).

Este argumento tiende a apoyarse en las supuestas experiencias de la segunda y tercera ola de inmigrantes europeos. De acuerdo con esta teoría, los inmigrantes europeos cortaron todo tipo de vínculos con su país de origen desde la primera generación y se integraron por completo a la vida política estadounidense. Sin embargo, los estudios sobre política transnacional indican que esto no es más que un mito.

Al contrario de lo que se cree, muchos inmigrantes europeos (los húngaros entre ellos) mantuvieron vínculos con el país de origen (Fitzgerald, 2000; Glick Schiller, 1999) y, aún así, se incorporaron a la vida política estadounidense. «A 
principios del siglo xx, los italianos y los judíos, al igual que muchos inmigrantes contemporáneos, seguían interesados y ávidamente involucrados en la vida política de su país de origen» (Foner 2000, 357). Hoy en día, además de los italianos y judíos, inmigrantes y no inmigrantes, hay grupos con ancestros polacos, irlandeses y de otros países que mantienen vínculos políticos con la tierra de origen. Incluso hay individuos que presentan candidaturas en el país natal, como los italo-americanos que intentaron obtener puestos parlamentarios en las elecciones italianas del 2006. La elevada participación de la comunidad judía en la política estadounidense a pesar de su constante interés político en Israel (Shain and Barth, 2003) es, sin duda, uno de los casos que mejor ilustran la viabilidad de la participación política binacional. Todos estos casos sugieren que el transnacionalismo no necesariamente obstaculiza la participación política a nivel nacional y que, de hecho, puede tener un efecto positivo y complementario.

Algunos estudios llevados a cabo en la década de los noventa, sobre todo en sociología, han examinado el aumento global en la frecuencia e intensidad de prácticas transnacionales (políticas, económicas y sociales) en algunas diásporas contemporáneas, entre ellas las de latinoamericanos, caribeños (Smith y Guarnizo, 1998) y mexicanos (Fitzgerald, 2000; Smith, 1998) residentes en Estados Unidos. Aunque estos trabajos no suelen explorar los efectos del transnacionalismo político en la participación política estadounidense o el nexo entre estos dos contextos, la información recabada no sugiere que el transnacionalismo lleve a una disminución en la participación nacional de quienes lo practican (Cano, 2002; Martínez-Saldaña, 2002; Moctezuma, 2003; Zabin y Escala-Rabadán, 1998). Por ejemplo, Rivera-Salgado (1999) menciona que, en California, ciertas organizaciones de migrantes indígenas originarios del estado de Oaxaca «han desarrollado un acercamiento binacional a la acción política» y construido alianzas con otros tipos de organizaciones de base estadounidenses para defender los derechos laborales y humanos de sus miembros en ese país. Este tipo de investigaciones nos llevan a echar un vistazo más cercano al nexo entre el ejercicio del transnacionalismo y la participación política a nivel nacional.

La información disponible muestra que los transnacionalistas son uno de los grupos menos proclives a distanciarse del sistema político estadounidense ya que, por lo general, poseen características socioeconómicas y sociodemográficas asociadas a mayores niveles de participación política. Los transnacionalistas latinoamericanos tienden a estar bien establecidos y asimilados (DeSipio et al., 2003; Escobar, 2004; Glick Schiller, 1999; González-Gutiérrez, 1995; Guarnizo et al., 2003; Smith, 1998), en especial aquellos afiliados a asociaciones de migrantes (Portes et al., 2007). Su sueldo, educación y nivel ocupacional suelen encontrarse por arriba del promedio; también suelen contar con ciudadanía o residencia permanente y han vivido en el país por largo tiempo. Sin embargo, hay que notar que los transnacionalistas mexicanos en específico -en particular los indígenas (Zabin y Escala-Rabadán, 1998) - suelen tener un perfil mucho menos privilegia- 
do. Estos datos sirven para explicar, al menos parcialmente, el porqué es tan improbable que los transnacionalistas se distancien de los procesos políticos estadounidenses, independientemente de su transnacionalismo.

Los estudios sugieren que el transnacionalismo no es un obstáculo para la participación política nacional y, quizá a pesar del perfil socioeconómico, puede incluso nutrirla. Las investigaciones sobre el papel de la doble nacionalidad sugieren que ésta facilita la participación política en Estados Unidos al incrementar los índices de naturalización, como ha ocurrido con los colombianos (Escobar, 2004) y otros latinoamericanos (Jones-Correa, 2001). Estudios sobre otras formas de transnacionalismo muestran que «el apego al país natal no disminuye la probabilidad de que los latinos participen en una variedad de actos políticos [en los Estados Unidos]» (Hritzuk y Park, 2000, 165). La información sobre colombianos, salvadoreños y dominicanos recabada por Guarnizo et al. (2003) indica que el transnacionalismo incrementó su autoestima y la confianza en su eficacia, lo cual muy probablemente incremente sus capacidades políticas en Estados Unidos. Portes (2003) enfatiza que la participación en actividades políticas transnacionales puede otorgar poder participativo a los inmigrantes y brindarles un objetivo $y$ autoestima que de otra manera no hubieran tenido (389). Una encuesta llevada a cabo por McCann et al. (2007) mostró que el ponerle atención a los asuntos mexicanos no disminuye la atención que le brindan los inmigrantes mexicanos al gobierno y elecciones estadounidenses, sino que incluso pueden incrementar sus posibilidades de participación (21). El sondeo de DeSipio et al. (2003) sobre puertorriqueños e inmigrantes mexicanos, salvadoreños y dominicanos indica que el transnacionalismo, más que las variables demográficas, juega un papel importante en la participación política en Estados Unidos. De hecho, los transnacionalistas estudiados mostraron una mayor inclinación a involucrarse en la vida política y cívica estadounidense que aquellos inmigrantes no transnacionales. También resultó más probable que se involucraran políticamente en Estados Unidos y no en su país de origen. Es por esto que DeSipio y sus colegas (2003) sugieren que las habilidades políticas aprendidas en el contexto transnacional son transferibles al contexto doméstico. Es decir, no existe ninguna contradicción entre el transnacionalismo y la participación política en Estados Unidos.

Existen otros estudios que sostienen que los inmigrantes latinoamericanos suelen participar en asociaciones de voluntarios y que su membresía en organizaciones transnacionales alienta su participación en la política estadounidense. Históricamente, estos grupos han creado y se han unido a organizaciones sociales, filantrópicas y políticas. De acuerdo con Fitzgerald (2000), durante décadas y sobre todo a partir de los noventa, los inmigrantes mexicanos han establecido asociaciones de ciudades locales o hometown associations (HTAS) y federaciones estatales conformadas por diversas HTAs. Al igual que los landsmanshaftn judíos (Foner, 2000), las HTAs son clubes sociales organizados por gente que proviene del mismo lugar. De acuerdo con varios autores (Fitzgerald, 2000; Goldring, 2002; 
González-Gutiérrez, 1995; Smith, 1998), la membresía en una HTA permite a los inmigrantes mantener vínculos religiosos, cívicos, culturales, económicos y políticos con sus lugares de origen. Y, aunque suene paradójico, la información recabada indica que estas afiliaciones también les permiten integrarse a la vida estadounidense de varias formas. Escobar (2004) documenta el caso de organizaciones colombianas en Nueva York que promueven la naturalización y ofrecen clases para obtener la ciudadanía. González-Gutiérrez (1995) reporta que las organizaciones mexicanas hacen cabildeo a favor de los inmigrantes y ofrecen cierto grado de representación política a nivel local:

Las actividades de los clubes y federaciones no se limitan a facilitar la llegada de nuevos inmigrantes a California; también representan y articulan las demandas de sus miembros... ante la sociedad civil y las autoridades locales de las ciudades en donde viven. (61)

Un estudio sobre las organizaciones salvadoreñas en Los Ángeles y Washington, D.C. da cuenta de cómo éstas educan a sus miembros sobre asuntos comunitarios como la inmigración, la política educativa y sus derechos en Estados Unidos (Landolt et al., 2003). De la Garza y Hazan (2003) reportan que las organizaciones de inmigrantes inculcan valores políticos estadounidenses a sus miembros, y datos de sondeos muestran que los transnacionalistas afiliados a este tipo de organizaciones son más propensos a involucrarse en las elecciones estadounidenses que los no afiliados (DeSipio et al., 2003). La afiliación ciertamente puede caracterizarse como un tipo de actividad transnacional (Guarnizo et al., 2003) y contribuye a la participación de los transnacionalistas en la vida política estadounidense.

De hecho, hay autores que sostienen que los inmigrantes mexicanos están cada vez más involucrados en la sociedad civil estadounidense y que el transnacionalismo ha jugado un papel importante en este proceso. Las actas de una conferencia llevada a cabo en el Woodrow Wilson Center en noviembre de 2005 dan muestra del grado de convicción de los participantes, quienes sostienen que si bien los inmigrantes se mantienen "simultáneamente comprometidos con la sociedad mexicana," también "contribuyen a los esfuerzos cívicos y políticos de la sociedad estadounidense» (Bada et al., 2006, v). Jonathan Fox define este proceso como el camino a una «binacionalidad cívica,»... un compromiso activo en ambos países» (Bada et al., 2006, vi).

Sin embargo, todavía hay huecos en la bibliografía académica sobre la transnacionalidad y sus efectos en la política estadounidense. Una de las preguntas que aún no se ha explorado es hasta qué nivel ayuda el transnacionalismo a desarrollar habilidades cívico-políticas que puedan utilizarse en el proceso político estadounidense. Y aunque se han hecho sondeos sobre inmigrantes latinoamericanos involucrados en acciones partidistas transnacionales y electorales (DeSipio et al., 2003) y la política en los países de origen (McCann et al., 2007), no hay estudios 
de caso sobre aquellos involucrados en otros tipos de actividades transnacionales como el cabildeo. Este trabajo busca llenar dichos huecos.

\section{PROPUESTA DE INVESTIGACIÓN}

Basándome en la información mencionada y la bibliografía sobre participación política existente, propongo que el transnacionalismo no obstaculiza la participación inmigrante en la política estadounidense ya que ofrece la oportunidad de desarrollar una serie de recursos -especialmente habilidades y práctica cívico-políticas- que resultan esenciales para la práctica política en cualquiera de las dos naciones. Mi propuesta general se basa en la premisa sugerida por Verba et al. (1995): mientras más involucrados estén los individuos, más fácil será su participación dada la experiencia acumulada. Como corolario, la experiencia política adquirida en un contexto puede transferirse a otro. De hecho, la hipótesis de transferencia de recursos propuesta por DeSipio et al. (2003) e incorporada en este estudio parte de esa idea. También me baso en el modelo de participación política desarrollado por Brady et al. (1995) para explorar este aspecto: se propone que aquello que aprenden los miembros de asociaciones civiles de voluntarios (por ejemplo, clubes sociales o asociaciones filantrópicas) no sólo es útil sino fundamental en el proceso de participación política. La membresía en este tipo de organizaciones ofrece la oportunidad de desarrollar habilidades cívicas esenciales para la participación política (toma de decisiones, voto, comunicación y organización, entre otras), incrementando así la posibilidad de un compromiso político. Es en base a estos conceptos teóricos que propongo que el transnacionalismo brinda la oportunidad de desarrollar recursos que también pueden ser utilizados en el contexto estadounidense.

Mis investigaciones ciertamente sugieren que los transnacionalistas desarrollan otros tipos de recursos que también pueden transferirse de un contexto a otro. Por ejemplo, las organizaciones transnacionales son poderosas herramientas de movilización política. Las redes de correo y comunicación electrónica se han convertido en un medio esencial de comunicación entre los transnacionalistas (latinos y de otros orígenes) y también facilitan la movilización política de simpatizantes en ambos lados de la frontera. Este artículo, sin embargo, se centra exclusivamente en la experiencia y habilidades cívico-políticas de los transnacionalistas mexicanos involucrados en la campaña a favor del voto ausente.

\section{PARTICIPACIÓN MEXICANA EN EL TRANSNACIONALISMO:}

EL CASO DE LA CAMPAÑA A FAVOR DEL VOTO AUSENTE (1998-2006)

El estudio de los transnacionalistas mexicanos involucrados en la campaña a favor del voto ausente nos brinda una excelente oportunidad de recabar nuevos datos 
y evidencia que corroboren o desmientan hallazgos previos sobre el impacto del transnacionalismo. Hasta ahora, esta campaña también representa el caso mexicano más importante de acción transnacional colectiva (efectuada tanto en México como en Estados Unidos) en pro de un objetivo particular. El proceso bien se puede describir como exigente dada la enorme oposición a la implementación del voto ausente en México y el hecho de que la campaña duró más de ocho años, necesitando viajes internacionales y una gran variedad de actividades políticas. Por lo tanto, resulta útil para identificar los tipos de habilidades cívico-políticas adquiridas por los transnacionalistas, evaluar el grado al que pueden llegar a transferirlas al terreno estadounidense y cómo es que lo hacen. También nos permite explorar si es cierto que el transnacionalismo distrae a los inmigrantes mexicanos de los eventos y procesos estadounidenses o si, al contrario, nutre y complementa su participación en la política doméstica. Si bien otros autores han documentado éste y otros casos de participación política mexicana (Martínez-Saldaña, 2002, 2003; Rodríguez-Oceguera, 2005) y el proceso legislativo en relación al voto ausente (Ross y Martínez-Cossio, 2004; Martínez-Saldaña y Ross-Pineda 2002), este trabajo es único en el sentido de que se enfoca en las implicaciones de dichos procesos en la participación política de los inmigrantes en territorio estadounidense.

El hecho de que la población de origen mexicano tradicionalmente se haya caracterizado por su bajo nivel de participación política (de la Garza, 2004) enfatiza la importancia de este estudio para activistas y legisladores, sobre todo si tomamos en cuenta que, con un total de 28.4 millones de personas en 2006, la diáspora mexicana representa la porción más amplia (64.1\%) del total de la población latina en Estados Unidos (44.3 millones). Lo que es más, una buena parte (40.1\%) comprende a los nacidos en México, y los inmigrantes muestran una mayor predisposición a practicar el transnacionalismo que los nacidos en Estados Unidos. Aún así, hay que aclarar que los transnacionalistas comprenden un porcentaje muy reducido de las poblaciones inmigrantes mexicana y latinoamericana en general (DeSipio et al., 2003; Guarnizo et al., 2003). En resumen, esta investigación busca contribuir, si bien de manera reducida, al entendimiento teórico y empírico de los efectos del transnacionalismo y la participación política de los inmigrantes mexicanos en la vida política estadounidense.

\section{PERFIL DE LOS TRANSNACIONALISTAS INVOLUCRADOS \\ EN LA CAMPAÑA A FAVOR DEL VOTO AUSENTE}

La campaña a favor del voto ausente duró ocho años e incluyó la participación de mexicanos residentes en Estados Unidos (la mayor parte de ellos inmigrantes de primera generación) y sus organizaciones, quienes se dieron a la tarea de persuadir a legisladores y dos presidentes mexicanos de implementar leyes que reconocieran el derecho al voto ausente. Entre 1998 y 2006, estos grupos participaron 
en una serie de actividades con el objeto de influir en los procesos políticos mexicanos; la Coalición por los Derechos Políticos de los Mexicanos (CDPME) se encontraba a la cabeza de las diversas organizaciones involucradas a nivel nacional. Fundada por inmigrantes mexicanos en 2001, la CDPME se convirtió en una coalición virtual de transnacionalistas a favor de voto ausente. De hecho, algunos de ellos habían promovido esta idea desde mediados de los noventa. De acuerdo con Primitivo Rodríguez-Oceguera, el coordinador de la cDpme en la Ciudad de México, la membresía en la organización ha variado de manera considerable. A mediados de la década del 2000 contaba con casi 400 afiliados individuales y 68 líderes de organizaciones sociales provenientes de 19 estados estadounidenses como California, Illinois, Texas y Arizona, además de una docena de simpatizantes en México (PRO, 2003, 2006). * La mayor parte de los afiliados eran activistas comunitarios y laborales o líderes de HTAS y federaciones, pero también se contaba con empresarios, profesionales, periodistas y académicos. Como se verá más adelante, estas identidades suelen reunirse en un solo individuo. Lo que es más, la mayor parte de ellos tiene el perfil socioeconómico y demográfico de otros transnacionalistas mexicanos y cuenta con una amplia cultura política. Por ejemplo, Raúl Ross-Pineda, el co-fundador de la cDPme y el líder de la campaña en la Ciudad de México, ha vivido en Chicago por casi 30 años. Es un analista político y escritor, ex-activista laboral y director fundador de la revista MX Sin Fronteras (RRP, 2003-2006). ${ }^{3}$

Muchos de los individuos que participaron en la campaña por el voto ausente (ya sea a través de la CDPME u otros grupos) estaban afiliados, de manera formal o informal, a una o más asociaciones transnacionales. Estas se pueden catalogar como asociaciones civiles comunitarias que sin embargo cuentan con una dimensión política dado el papel que juegan como representantes de los intereses de sus miembros en Estados Unidos y México, sus actividades transnacionales, y el apoyo que le brindaron a la campaña a favor del voto ausente. Entre ellas se incluyen las HTAS y federaciones, organizaciones comunitarias, y grupos de trabajadores inmigrantes. Por ejemplo, además de ser uno de los miembros principales de la CDPME, Luis Pelayo lidera el Concilio Hispano, una organización comunitaria con sede en Chicago. Otro ejemplo es Florencio Zaragoza, afiliado de la CDPME y dirigente de la Fundación México, una organización sin ánimo de lucro fundada en 1998 en Tucson, Arizona. Su trabajo involucra representar a los inmigrantes y promover asuntos, servicios comunitarios a nivel local, estatal, nacional y transnacional.

Otros transnacionalistas como José Luis Gutiérrez pertenecieron, en un momento u otro, a HTAs creadas con metas transnacionales sociales y filantrópicas. Su función es recaudar remesas colectivas entre los miembros para financiar proyectos de infraestructura como potabilización del agua, construcción de escuelas,

* Las iniciales señaladas entre paréntesis en el texto restante de este artículo, se refieren a las personas con las que tuve comunicaciones personales/entrevistas. Sus nombres completos y perfiles se describen en el Apéndice A.

3 Creada por inmigrantes mexicanos en 2005, esta revista (publicada en Chicago hasta 2007) exploraba asuntos binacionales y temas relacionados con los inmigrantes en Estados Unidos. 
cementerios, campos de fútbol, caminos, o clínicas de salud en las comunidades de origen (Goldring, 2002; González-Gutiérrez, 1995; Smith, 1998). Como consecuencia del papel que juegan en el abasto y distribución de los fondos para obras públicas, las HTAs y sus líderes cobran importancia política a nivel local, municipal, estatal e incluso nacional en el país de origen, aún cuando hayan sido creadas como clubes sociales. Un ejemplo es el Club Morelia de Chicago, fundado por inmigrantes provenientes de esa ciudad michoacana.

Algunos de los transnacionalistas mexicanos involucrados en la campaña provenían de organizaciones con fines políticos intrínsecos tales como las federaciones, frentes o consejos. Éstos representan el brazo político de muchas HTAs y grupos asociados. Las federaciones representan a HTAs del mismo Estado mexicano y a veces se identifican a partir del Estado estadounidense en el cual se encuentran, como es el caso de la poderosa Federación de Clubes Michoacanos en Illinois (FEDECMI). Los frentes comprenden asociaciones de inmigrantes que vienen de un mismo estado y viven en regiones específicas de Estados Unidos, como en el caso del Frente Binacional de Michoacanos (FreBIMICH), una organización cívico-política creada en 2004 para representar a los michoacanos residentes en Illinois, Wisconsin, Indiana, Nevada, Texas, California y otros estados. Las organizaciones de origen indígena representan otro tipo de frente y sus miembros suelen ser originarios del estado de Oaxaca, como en el caso del Frente Indígena de Organizaciones Binacionales (FIOB, antes Frente Indígena Oaxaqueño Binacional). Este grupo con sede en Fresno, California, se encontraba liderado por Rufino Domínguez durante la campaña a favor del voto ausente (para más sobre estas organizaciones véase Rivera-Salgado, 2002). Los consejos o confederaciones suelen incluir a varias federaciones de distintos estados mexicanos asentadas en una región estadounidense específica. Dos ejemplos son el Consejo de Federaciones Mexicanas en Norte América (COFRem), el cual tiene su sede en Los Ángeles y representa federaciones de 16 estados mexicanos, y la Confederación de Federaciones de Mexicanos del Medio Oeste (Confemex), con sede en Illinois.

Sin embargo e independientemente del lugar de origen de sus miembros, a partir de 1998 se crearon una serie de organizaciones específicamente dedicadas a la campaña por el voto ausente. Algunas, como la CDPME, siguen existiendo; otras desaparecieron o se han integrado a otras asociaciones. Entre ellas se incluyen la Coalición de Mexicanos en el Exterior Nuestro Voto 2000; la Coalición Internacional de Mexicanos en el Exterior (CIME), con miembros en Texas, Chicago, Nueva York y Nueva Jersey; el Consejo Electoral Mexicano del Medio Oeste; el Comité Nacional Pro Voto México 2000; la Campaña Nacional por el Voto Ausente; Migrantes Mexicanos por el Cambio (MIMEXCA), fundada por líderes empresariales en California en 1998, y la Organización de Mexicanos en el Exterior (OEM), fundada en 2003.

Una organización bastante peculiar que también puede ser catalogada como transnacional y política es el Consejo Consultivo del Instituto de los Mexicanos 
en el Exterior (CC-IME). Dicho cuerpo está altamente institucionalizado y a cargo del gobierno mexicano (Cano y Délano, 2004). El IME, una oficina para asuntos relacionados con los migrantes dentro de la Secretaría de Relaciones Exteriores (SRE), fue creado en 2002 por el presidente Vicente Fox y bajo presión de los transnacionalistas mexicanos residentes en Estados Unidos. El CC supervisa al IME y está compuesto por 100 transnacionalistas que fungen como representantes de la diáspora y aconsejan al gobierno mexicano. La participación en el CC-IME es voluntaria y la mayor parte de sus miembros deben competir para ser nombrados y electos por las comunidades de origen mexicano a lo largo de Estados Unidos. ${ }^{4}$ Si bien los miembros del CC-IME cabildearon a favor del voto ausente como miembros de otras coaliciones, también se manifestaron al respecto como representantes de la comisión de asuntos políticos del instituto (resulta interesante que, en un momento dato, el gobierno mismo haya sido uno de sus blancos, ya que Fox, no parecía ser capaz de tomar una postura clara a favor de la nueva legislación, o al menos no estaba dispuesto a hacerlo). En general, estas distintas organizaciones, sus líderes y otros activistas se involucraron en diversos tipos de actividad política transnacional con el propósito de garantizar la participación de los expatriados mexicanos en las elecciones. La siguiente sección da cuenta del tipo de habilidades y experiencia que adquirieron al participar en dichas organizaciones y la campaña misma.

\section{EL TRANSNACIONALISMO FACILITÓ LA PARTICIPACIÓN EN LA POLIITICA ESTADOUNIDENSE}

Una de las consecuencias principales del transnacionalismo es que los involucrados adquirieron recursos esenciales para la participación política en ambos lados de la frontera. En específico, la participación en organizaciones transnacionales y la campaña por el voto ausente les permitió adquirir y utilizar habilidades cívicas y políticas. El Cuadro 1 muestras los dos tipos de participación (i.e., en organizaciones y actividades políticas) y el tipo de habilidades que, una vez desarrolladas, podrían utilizarse en el terreno estadounidense. Los miembros de organizaciones transnacionales tuvieron la oportunidad de aprender y/o utilizar las habilidades cívicas necesarias para la participación política (por ejemplo, la recolección de fondos, hablar en público, votar, dirigir reuniones y tomar decisiones). En algunos casos hasta podían recibir cursos en liderazgo brindados por su organización. Lo que es más, la campaña a favor del voto ausente les permitió desarrollar sus habilidades en el exigente contexto de la política nacional y más allá de los límites de los asuntos comunitarios. Los transnacionalistas ahora cuentan con mucho más experiencia y sofisticación política, independientemente del país en el que participen. Las siguientes secciones muestran cómo es que adquirieron dichas habilidades y cómo es que esto ha afectado su participación en la política estadounidense. 


\section{CUADRO 1}

De cómo la participación en el transnacionalismo facilita la participación en la política estadounidense

\begin{tabular}{|c|c|}
\hline TIPO DE PARTICIPACIÓN TRANSNACIONAL & $\begin{array}{l}\text { MANERAS EN QUE LA PARTICIPACIÓN TRANSNACIONAL BENEFICIA } \\
\text { LA PARTICIPACIÓN EN LA POLIÍTICA ESTADOUNIDENSE }\end{array}$ \\
\hline \multicolumn{2}{|l|}{$\begin{array}{l}\text { Participación en organizaciones transnacionales } \\
\text { como HTAS mexicanas, federaciones de HTAS } \\
\text { y otras asociaciones civiles o politicas }\end{array}$} \\
\hline $\begin{array}{l}\text { Los transnacionalistas adquieren y/o practican ha- } \\
\text { bilidades cívicas como miembros de dichas orga- } \\
\text { nizaciones. }\end{array}$ & $\begin{array}{l}\text { Las habilidades cívicas son necesarias para la par- } \\
\text { ticipación política en cualquier país (ya sea de ori- } \\
\text { gen o residencia). }\end{array}$ \\
\hline $\begin{array}{l}\text { Las habilidades cívicas incluyen: la organización y } \\
\text { dirección de encuentros; recaudación de fondos } \\
\text { y otros eventos; la comunicación escrita; hablar en } \\
\text { público; votar; dirigir gente y organizaciones; hacer } \\
\text { donaciones, y persuadir a otros de apoyar ciertos } \\
\text { asuntos. }\end{array}$ & $\begin{array}{l}\text { Los transnacionalistas que cuentan con habilidades } \\
\text { cívicas están mejor preparados y dispuestos a par- } \\
\text { ticipar en eventos políticos como elecciones, cam- } \\
\text { pañas, movilización de grupos de base, o candidatu- } \\
\text { ras en cualquier país. }\end{array}$ \\
\hline \multirow[t]{2}{*}{$\begin{array}{l}\text { Las habilidades cívicas son las mismas que aquellas } \\
\text { requeridas para la participación política. }\end{array}$} & $\begin{array}{l}\text { Ejemplo: los líderes de las federaciones de HTAs ne- } \\
\text { gocian con alcaldes y gobernadores mexicanos en lo } \\
\text { que respecta a la distribución de las remesas desti- } \\
\text { nadas a obras públicas en pueblos mexicanos. }\end{array}$ \\
\hline & $\begin{array}{l}\text { Las habilidades que han adquirido sirven para par- } \\
\text { ticipar en las políticas mexicana y estadounidense. } \\
\text { Los transnacionalistas han usado habilidades simil- } \\
\text { ares en la campaña por la reforma inmigratoria. }\end{array}$ \\
\hline \multicolumn{2}{|l|}{$\begin{array}{l}\text { Participación en actividades políticas transnacionales } \\
\text { (por ejemplo, la campaña por el voto ausente en México) }\end{array}$} \\
\hline $\begin{array}{l}\text { Los transnacionalistas adquieren y/o practican ha- } \\
\text { bilidades políticas. }\end{array}$ & $\begin{array}{l}\text { Las habilidades políticas aprendidas y/o practicadas } \\
\text { durante las actividades transnacionales pueden utili- } \\
\text { zarse para participar políticamente en cualquier país. }\end{array}$ \\
\hline \multirow{2}{*}{$\begin{array}{l}\text { Estas incluyen: votar; hacer propuestas políticas; } \\
\text { hablar con los votantes; donar a campañas políti- } \\
\text { cas; movilizar grupos de base para persuadir a los } \\
\text { legisladores de apoyar ciertas medidas; ejercer influ- } \\
\text { encia; negociar y cabildear con representantes gu- } \\
\text { bernamentales y exigirles cuentas claras. }\end{array}$} & $\begin{array}{l}\text { Ejemplo: los transnacionalistas mexicanos desarrol- } \\
\text { laron habilidades políticas a raíz de su participación } \\
\text { en la campaña por el voto ausente en México (1998- } \\
\text { 2006). }\end{array}$ \\
\hline & $\begin{array}{l}\text { Han utilizado habilidades similares en Estados Uni- } \\
\text { dos en casos como el de la reforma inmigratoria. }\end{array}$ \\
\hline
\end{tabular}

\section{La participación en organizaciones transnacionales ayudó} a desarrollar habilidades cívico-políticas necesarias para la participación política en Estados Unidos

Guadalupe Gómez nació en Jalpa, Zacatecas, pero ha vivido en Orange County, California, por más de 30 años. De origen rural, eventualmente estableció su propio negocio y adquirió prestigio como líder comunitario a nivel local, estatal y nacional, tanto en México como en Estados Unidos. Comenzando como el secretario del Club Deportivo Santa Juana (HTA), Gómez desarrolló sus habilidades organizando encuentros y recaudación de fondos, estableciendo comunicación y 
presentándose como candidato (GG, 2003, 2006). Con el tiempo obtuvo cargos más altos y llegó a ocupar varios puestos en la poderosa FCZSC, de la cual fue presidente de 2000 a 2005. También fue presidente del cofrem en 2002 y es co-fundador del Frente Cívico Zacatecano (FCZ) (GG 2006; Moctezuma, 2003). Es gracias a este tipo de experiencias que Gómez se ha entrenado en actividades como el cabildeo y la negociación con representantes municipales, estatales y federales en relación a la distribución de las remesas en el Programa 3x1 de Zacatecas. ${ }^{5}$ Aprendió a comunicarse con representantes gubernamentales mexicanos para tratar asuntos locales y también a actuar como intermediario entre algunos de ellos y sus contrapartes estadounidenses en relación a asuntos como comercio y otro tipo de intercambios. La membresía en el CC-IME, al cual se unió en 2003, le ha permitido adquirir aún más experiencia y recursos como actor político.

Ciertamente, la membresía en el CC-IME permite que los transnacionalistas refinen sus habilidades políticas, incluyendo su capacidad de organización y liderazgo. Ya que el trabajo de sus miembros consiste en aconsejar al gobierno mexicano sobre política migratoria, es necesario que estén informados con respecto a los asuntos comunitarios, hablen con sus electores, analicen y discutan políticas públicas alternativas, dirijan grupos de trabajo, negocien acuerdos y presenten propuestas. Estas actividades contribuyen a su politización y capacidad de movilización y liderazgo en ambos lados de la frontera. De hecho y de acuerdo con algunos representantes del IME (CGG, 2003, 2006; GC, 2006), una de las metas principales del instituto es entrenar a los individuos en el desempeño de estas tareas. La estructura operativa del CC-IME, sobre todo los encuentros organizados regularmente en México y otros lugares, constituyen un espacio en donde consejeros de distintas partes de México y Estados Unidos que quizá no se conocían antes pueden reunirse a discutir e intercambiar ideas u organizar actos políticos en cualquiera de las dos naciones.

Los beneficios de pertenecer a asociaciones transnacionales suelen verse acrecentados ya que los transnacionalistas como Gómez pueden participar, ya se de forma simultánea o consecutiva, en varias organizaciones. Como indiqué antes, muchos de los involucrados en la campaña a favor de voto ausente se encontraba afiliados a más de una organización (por ejemplo, el Concilio Hispano, el CC-IME y la CDPME); al menos diez de los líderes y activistas afiliados a la CDPME también aparecían como consejeros del CC-IME en la junta del 2003-2005.

\section{La participación en la campaña a favor del voto ausente ayudó a desarrollar habilidades esenciales para participar en la política estadounidenses}

Después de la eliminación de los obstáculos constitucionales que impedían el voto ausente en 1996, el proceso para implementar el voto mismo se paralizó en

\footnotetext{
5 Bajo este programa, cada nivel gubernamental mexicano contribuye un dólar por cada dólar enviado por los migrantes y los fondos se destinan a obras públicas (Goldring, 2002).
} 
México, así que los transnacionalistas comenzaron a organizar una campaña de cabildeo en 1997 (Martínez-Saldaña y Ross-Pineda, 2002). Muchos de ellos estaban haciendo cabildeo por primera vez en sus vidas y tuvieron que aprender conforme avanzaba el proceso. La campaña de ocho años les permitió aprender tácticas políticas esenciales como

- la construcción de coaliciones políticas;

- la presentación de sus intereses ante comités legislativos, miembros del gabinete y partidos políticos;

- la organización de campañas de base para, a través de cartas y correos electrónicos, convencer a representantes políticos de apoyar el voto ausente;

- la organización de eventos públicos para movilizar simpatizantes;

- brindar nueva información y redactar propuestas legislativas para influir en la implementación de leyes;

- escribir artículos de opinión en los periódicos;

- asediar a los representantes pertinentes en eventos públicos, los pasillos del Congreso o sus oficinas para convencerlos de apoyar medidas particulares. ${ }^{6}$

En resumen, la campaña permitió que los transnacionalistas adquirieran y/o practicaran habilidades cívico-políticas que también resultan útiles en el contexto estadounidense. Algunos ejemplos concretos se presentan a continuación.

Entre 1998 y 2005 los transnacionalistas organizaron docenas de foros y encuentros en ambos países con el objeto de lograr tres metas: 1) informar a otros inmigrantes, simpatizantes, oponentes, académicos y representantes del gobierno mexicano sobre el voto ausente y la representación política de los migrantes en México, 2) iniciar un debate al respecto, y 3) movilizar grupos de base (Martínez-Saldaña, 2003). El primer foro fue patrocinado por la Campaña por el Voto Ausente en el 2000 (un predecesor de la CDPME) y se llevó cabo en Sacramento, California, en $1998 .^{7}$

Los transnacionalistas también tuvieron que cortejar y presionar a los representantes gubernamentales mexicanos en ambos lados de la frontera. Por ejemplo, en junio del 2001, la Coalición Internacional de Mexicanos en el Extranjero (CIME, otro predecesor de la CDPME) organizó un evento en Chicago para convencer a legisladores mexicanos de que presentaran el caso del voto ausente ante sus comisiones parlamentarias (Ross y Martínez-Cossio, 2004). Mientras tanto, algunos representantes individuales como Ross-Pineda, Luis de la Garza y Carlos Olamendi viajaban constantemente a la Ciudad de México para cabildear y trabajar con representantes del gobierno mexicano, incluyendo al entonces Subsecretario de Desarrollo José Francisco Paoli (SEGOB); representantes de los principales partidos políticos y el Instituto Federal Electoral (IFE), y legisladores en comisiones clave, como la Comisión de Asuntos Fronterizos Norte del Senado. El propósito

6 Para más detalles sobre las prácticas de cabildeo, véanse Schmidt et al. (2008).

7 Yo estuve presente en algunos de estos eventos a partir del encuentro en Los Ángeles en 2003.

102 PRIMER SEMESTRE 2009 MIGRACIÓN Y DESARROLLO 
era brindarles información sobre las preferencias y propuestas políticas y legales de los expatriados.

Otros actores importantes se concentraron en promover la iniciativa del voto ausente en sus estados de origen. Jesús Martínez-Saldaña, líderes del FrEBIMICH y la FEDECMi como José Luis Gutiérrez, y líderes de la FCZSC y el fCZ como Gómez, redactaron propuestas legislativas que presentaron a los gobernadores y legisladores estatales. Los transnacionalistas también llevaron delegaciones de expatriados a cabildear en México. Olamendi, por ejemplo, llevó un grupo de unas veinte personas a que se reunieran con Fox, miembros de su gabinete, legisladores, y representantes de los distintos partidos y del ife en la Ciudad de México en marzo de 2002 (CO, 2003; Cano, 2002).

Se implementaron también varias otras tácticas, incluyendo la redacción de propuestas legislativas presentadas ante el gobierno mexicano. En agosto de 2003 yo presencié una reunión en la Ciudad de México entre varios líderes de la CDPME (incluyendo a Ross-Pineda, Rodríguez y Martínez de la Garza) y el entonces Secretario de Gobernación, Santiago Creel, a quien se le entregó la más reciente propuesta legislativa. Además, los líderes de la CDPME escribieron numerosas cartas a las comisiones legislativas y, junto con el CC-IME y los líderes de federaciones, movilizaron a los expatriados mexicanos en Estados Unidos para que presionaran a determinados legisladores a través de cartas y recolección de firmas (por ejemplo, las cartas enviadas al Senado en marzo del 2005). También intentaron ejercer su influencia a través de los medios de comunicación, publicando artículos de opinión en los periódicos y dando seis conferencias de prensa a favor del voto ausente. Finalmente, después de ocho años de luchar contracorriente, sus esfuerzos rindieron resultado.

Gracias a la campaña, la nueva legislación fue aprobada a nivel federal en junio de 2005, permitiendo que los expatriados votaran en las elecciones presidenciales de 2006. Las campañas estatales también rindieron fruto en Michoacán (2007) y Zacatecas (2003), permitiendo a los expatriados de dichos estados participar en el proceso electoral. Queda claro que, para entonces y a pesar de la reticencia en el Congreso, la presidencia y los partidos políticos, los promotores del voto ausente habían adquirido suficiente experiencia para lograr que se implementara la legislación.

Una vez alcanzadas estas metas tuve la oportunidad de presenciar el impacto del transnacionalismo en la confianza política de algunos actores. En agosto de 2005, la CDPME organizó una ceremonia conmemorativa en un monumento nacional en la Ciudad de México. Acompañados por representantes del gobierno mexicano, legisladores, ciudadanos mexicanos y los medios de comunicación, los transnacionalistas se encontraban henchidos de orgullo al presenciar lo que describieron como un gran triunfo de los migrantes mexicanos. Sus discursos y comentarios se vieron acompañados por una gran seguridad y la noción de que no sólo podían sino también debían influir en los asuntos políticos en ambos lados de la 
frontera (PRO, RRP, JMS, 2005). Ciertamente, la confianza en su eficacia política parecía haberse incrementado.

Con renovado entusiasmo y nueva experiencia, los transnacionalistas mexicanos iniciaron campañas de promoción del voto ausente y también de participación política en general entre los mexicanos residentes en Estados Unidos: ahora querían influir en los resultados de las elecciones presidenciales de 2006. El COFREM, los consejeros del CC-IME, las federaciones, HTAS y otros grupos utilizaron los recursos adquiridos (habilidades, contactos, redes e infraestructura) para organizar campañas comunitarias a lo largo del país e informar a los expatriados sobre la nueva legislación, facilitar el registro de los votantes y promover el voto mismo. Más de 100 asociaciones mexicanas organizaron una docena de campañas para promover el voto ausente. En noviembre de 2005, la FCZSC, junto con otras federaciones y la cadena Telemundo, llevo a cabo campañas informativas y de registro en espacios públicos de Santa Ana y Los Ángeles, California, dos de los destinos más importantes de inmigrantes mexicanos en Estados Unidos (GG, 2005). Un día antes del 15 de enero de 2006, la fecha límite para registrarse en el padrón electoral y votar en la elección mexicana, el cofrem, la Federación Californiana de Michoacanos, la Federación Bajacaliforniana y otros grupos se apuraron a registrar al mayor número posible de inmigrantes (Truax, 2006a). Tan sólo un mes después, el COFReM y grupos asociados lanzaron otra campaña-Campaign for Unity and Fraternity- para movilizar a las federaciones e inmigrantes mexicanos a favor de los derechos de los migrantes (Truax, 2006b). Esta vez, sin embargo, intentarían influir en la política estadounidense.

\section{Los transnacionalistas utilizan sus habilidades}

para participar en la política estadounidense: la campaña

a favor de la reforma inmigratoria en la década del 2000

Mientras trabajaban en la campaña a favor del voto ausente y el proceso electoral mexicano, los transnacionalistas utilizaron las habilidades aprendidas para intentar influir en los procesos y asuntos estadounidenses. Desde principios de la década del 2000, Olamendi, de la Garza, Pelayo y otros miembros de la Latino Coalition habían redactado propuestas de reforma y trabajado con legisladores republicanos estadounidenses para promoverlas (CO 2003, 2006; LP, 2003). En mayo de 2003 y con el apoyo de la sede en Washington D.C. de la Latino Coalition, Olamendi organizó una conferencia en dicha ciudad con el propósito de renovar el interés por la reforma inmigratoria discutida por los presidentes de Estados Unidos y México antes de los atentados del 11 de septiembre de 2001 (Canto y Bunis, 2003). Para 2006, los líderes de la CDPME ya estaban discutiendo la necesidad de llegar a un consenso sobre la reforma inmigratoria en Estados Unidos para poder cabildear con legisladores estadounidenses. En mayo de 2006 
Olamendi y otros colaboradores organizaron un evento político en Irvine, California, que contó con la presencia del senador John McCain (R-Az). Terminado este evento, miembros de la CDPME y otros transnacionalistas se reunieron a discutir y revisar otra propuesta redactada en la primavera de $2006 .^{8}$ El borrador se circuló entre afiliados de la CDPME a través del correo electrónico para recibir su opinión y sugerencias.

Lo que es más, estos no eran los únicos transnacionalistas involucrados en la política estadounidense en esa época. Otros estaban organizando marchas y diversos eventos políticos. Como ejemplo podemos citar los cientos de marchas y mítines a favor de los derechos de los inmigrantes ocurridos entre febrero y mayo de 2006 en ciudades como Los Ángeles, Chicago y Dallas, por no mencionar aquellos llevados a cabo desde 2000. En 2006, estos mismos grupos apoyaron una reforma inmigratoria integral (es decir, centrada en la legalización de los inmigrantes indocumentados residentes en Estados Unidos) y protestaron contra la propuesta de ley de Sensenbrenner, aprobada por la Cámara de Representantes en diciembre de 2005. ${ }^{9}$ La Confemex y líderes como Fabián Morales, Luis Pelayo y José Artemio Arreola formaron el Comité 10 de marzo, el cual se convirtió en uno de los detonadores de los mítines y marchas que llevaron al movimiento proinmigrante de 2006 en Chicago (FM, 2006; JAA, 2006; LP, 2006). El Comité organizó varios eventos relacionados con la reforma inmigratoria, incluyendo marchas, conferencias de prensa, una serie de foros en junio del 2006 y una convención nacional en agosto del mismo año (JAA, 2006). Francisco Zamora-Horta de la Casa Guanajuato, con sede en Atlanta, planeó mítines, boicots y una campaña de movilización para animar a otros transnacionalistas a que se pusieran en contacto con representantes gubernamentales, los medios de comunicación e iglesias para apoyar una reforma inmigratoria integral (FZH, 2006). De la Garza participó en las audiencias del consejo ciudadano de Farmers Branch (Dallas, Texas) en contra de las leyes anti-inmigrantes (LDLG, 2006). También hay que mencionar a los miembros del FIOB, quienes salieron a las calles de California para manifestarse a favor de los derechos de los inmigrantes. Años más tarde, en 2009, los transnacionalistas nuevamente están utilizando sus habilidades para promover la reforma inmigratoria bajo la administración de Barack Obama.

Mientras los participantes en los mítines de 2006 clamaban «hoy marchamos, mañana votamos», algunos transnacionalistas organizaron campañas para promover la naturalización, el registro de votantes y el voto en Estados Unidos. La COFREM, la FCZSC y otros se unieron a la coalición We Are America (Nosotros somos América) liderada por el Service Employees International Union (Sindicato Internacional de Trabajadores del Servicio, SEIU) para promover la naturalización e influir en la creación de la política inmigratoria estadounidense (La Opinión, 2006). Esta coalición lanzó la campaña Summer for Democracy (Verano por la democracia) como parte de un esfuerzo nacional por incrementar la participación

8 Yo estuve presente en esta reunión.

9 Sensenbrenner negaba la legalización y pedía mayor control fronterizo y policiaco. 
cívica entre las comunidades inmigrantes en Estados Unidos. La National Alliance of Latin American and Caribbean Communities (Alianza Nacional de Comunidades Latinoamericanas y Caribeñas, NALACC), una coalición compuesta por 75 organizaciones de inmigrantes y fundada en conjunción con la FEDECMI, lanzó la campaña Familias Unidas a nivel nacional en marzo de 2005 (JLG, 2005). Su propósito es promover la reforma inmigratoria junto con la naturalización y la participación en Illinois, Nueva York, California, Massachussets, Texas y Florida. Las tácticas de la NALACC son similares a aquellas utilizadas en la campaña a favor del voto ausente e incluyen reunirse con miembros del Congreso y la Casa Blanca, organizar marchas, vigilias, reuniones caseras, conferencias de prensa, peticiones y recaudación de firmas, formar de alianzas y promover el registro y movilización de votantes, así como el envío de cartas, postales y faxes a los legisladores (JLG, 2005).

\section{LA CUESTIÓN DE LA TRANSFERENCIA}

Claramente, la transferencia de recursos también se manifiesta en el hecho de que algunos transnacionalistas han desarrollado habilidades cívico-políticas en otros contextos. En algunos casos éstas fueron adquiridos antes de la participación transnacional en procesos mexicanos o estadounidenses. Algunos miembros de la CDPME habían estado involucrados en la política desde antes de dejar México. Ross-Pineda, Olamendi, Domínguez y de la Garza, entre otros, habían fungido como líderes o activistas en sindicatos, movimientos sociales y/o partidos políticos mexicanos antes de mudarse a Estados Unidos. También hay otros cuya experiencia previa al transnacionalismo había involucrado activismo sindical o comunitario en Estados Unidos (Martínez-Saldaña, 2002) -Ross-Pineda, Arreola y Jorge Mújica son algunos de ellos. Gómez, por ejemplo, sostiene que «antes de participar en el transnacionalismo, él votaba en Estados Unidos» (JG, 2006). La evidencia de la campaña por el voto ausente sugiere que algunas de las tácticas que se utilizaron en el contexto transnacional fueron de hecho aprendidas en Estados Unidos. Algunas de las estrategias de cabildeo empleadas para persuadir a los legisladores mexicanos, como las campañas por medio de cartas y peticiones, son características del contexto estadounidense y poco comunes en México. Sin embargo e independientemente del lugar en donde se aprendieron estas habilidades, todo parece indicar que sirven para influir en los procesos políticos de ambos países.

\section{LA PARTICIPACIÓN DE LOS TRANSNACIONALISTAS EN LA POLÍTICA ESTADOUNIDENSE}

Aunque los transnacionalistas parecen estar bastante interesados en participar en la vida política estadounidense, dicha participación varía en forma y grado, como

106 PRIMER SEMESTRE 2009

MIGRACIÓN Y DESARROLLO 
se mostró en la sección anterior. Las HTAs promueven la integración política, pero algunas otras organizaciones cuentan con un grado de participación y sofisticación bastante alto. Algunas federaciones y frentes incluso se encuentran registrados como political action committees (comités de acción política, PACs) en Estados Unidos. El fCz, fundado por Gómez y sus colegas a finales de la década de los noventa como el brazo político de la FCZSC, se ha convertido en una PAC con la capacidad de apoyar candidatos tanto demócratas como republicanos que quieran postularse a nivel local, estatal e incluso nacional (GG, 2006; Moctezuma 2003). El frebiMich ha apoyado las campañas del congresista Luis Gutiérrez (D-IL) y representantes locales y estatales (JLG, 2004). Mexicans for Political Progress (Mexicanos a favor del progreso político, MXPP), una PAC con sede en Chicago formada recientemente por transnacionalistas como Fabián Morales, se creó justo a tiempo para apoyar la reelección del gobernador de Illinois, Rod Blagojevich (JLG, 2006).

La participación individual también varía en grado y tipo. La evidencia recabada por mi comunicación electrónica informal con algunos transnacionalistas en 2006 da cuenta de las diversas maneras en que se llevan a cabo estas contribuciones. Los naturalizados dijeron que participaban en las elecciones estadounidenses y otros procesos. Varios de los no ciudadanos mencionaron que se ponen en contacto con representantes gubernamentales y hacen cabildeo, participan en mítines y se involucran en actividades comunitarias y su organización. Todos prestan atención a las noticias y participan de manera regular en discusiones sobre la política en ambos países, muchas veces a través de medios electrónicos.

Algunos transnacionalistas participan de manera distinta. El líder de la CDPME, Ross-Pineda, participa de manera poco activa en grupos estadounidenses a pesar de su alto nivel de transnacionalidad (RRP, 2006). De hecho, en lugar de presentarse a sí mismo como un actor político en cualquiera de los dos países, Ross-Pineda prefiere definirse como un promotor de los derechos de los migrantes mexicanos en Estados Unidos. Por lo tanto, participó como activista en el debate sobre el Tratado de Libre Comercio de América del Norte (TLCAN) a principios de los noventa y ha trabajado con el American Friends Service Committee (Comité de Servicio Amigos Americanos) en Chicago. También participó en la elaboración de las propuestas hechas por la CDPME con respecto a la reforma migratoria en México y Estados Unidos. En sus diversos roles como analista político y escritor, director de la revista $M X$ Sin Fronteras y creador y administrador de redes electrónicas y un portal de Internet al servicio de la comunidad transnacional, Ross-Pineda ha contribuido a la toma de conciencia política binacional de los migrantes mexicanos.

Sin embargo, hay los que están sumamente involucrados a todos los niveles. Olamendi es un ejemplo. Tiene fuertes lazos con el partido republicano y ha apoyado campañas como las del gobernador de California Arnold Schwarzenegger en 2003 y el presidente George W. Bush en 2000. También hizo campaña por 
Vicente Fox en México en el 2000 (CO, 2003). Además de ser un miembro principal de la CDPME, Olamendi ha sido miembro y/o fundador de numerosas PACs y asociaciones políticas y empresariales, como la New Coalition for Comprehensive Immigration and Security Reform (Nueva Coalición a Favor de una Reforma Integral en Inmigración y Seguridad), además de haber trabajado de cerca con los senadores McCain y Ted Kennedy (D-Ma) en relación a este tema (CO, 2006). José Luis Gutiérrez, el líder de la FEDECMI, es otro ejemplo. Establecido en Chicago, funge como activista comunitario y ha participado en procesos electorales a nivel local, estatal y nacional (JLG, 2004). Fue miembro fundador de la NALACC en 2005 $y$, al mismo tiempo, se mantuvo involucrado en la campaña a favor del voto ausente. En abril de 2006 fue nombrado director de la Oficina de Asuntos de Inmigración del Gobernador de Illinois (JLG, 2006).

\section{ALGUNAS CONCLUSIONES}

Este estudio se propuso investigar cómo es que el transnacionalismo político afecta la capacidad de los transnacionalistas mexicanos de participar en la política estadounidense. Se propuso que el ejercicio del transnacionalismo contribuye al desarrollo de experiencia y habilidades cívico-políticas que son transferibles de un contexto a otro y que facilitan la participación en el terreno estadounidense. La participación de los transnacionalistas en organizaciones civiles y la campaña a favor del voto ausente corrobora parcialmente esta propuesta: se constata que desarrollaron y ejercieron actividades como el cabildeo, la organización, la comunicación y otro tipo de estrategias que posteriormente parecen haber implementado en Estados Unidos. Se puede decir, por lo tanto, que estas habilidades facilitaron e incluso incrementaron su capacidad de participación política en dicho país. Sus actividades en relación al debate sobre la reforma inmigratoria en Estados Unidos sugieren que las habilidades y experiencia adquiridas también fueron implementadas en el contexto estadounidense.

Esta investigación contribuye de manera modesta pero importante a la literatura sobre el tema. La evidencia corrobora resultados previos con respecto al capital humano adquirido por los inmigrantes que ejercen el transnacionalismo y su mayor nivel de participación política. Lo que es más, la información recabada confirma que el transnacionalismo es una variable crucial en la participación política. No encontré evidencia alguna sobre el posible desinterés de los transnacionalistas mexicanos en la política de su nueva patria, como argumentan algunos críticos. Tampoco hay nada que sugiera que el poder político de la comunidad latina sufrirá pérdidas a raíz del transnacionalismo. Al contrario, los transnacionalistas no difieren mucho del resto de los estadounidenses: mientras más expuestos a procesos políticos estén y más involucrados en estos se vean, más interesados estarán en participar, independientemente del país. 
Estos datos pueden ser de ayuda para legisladores y activistas que tienen duda con respecto a las prácticas transnacionales. El transnacionalismo sirve como una herramienta educativa que contribuye a los esfuerzos de la comunidad latina por incrementar su participación política en Estados Unidos. En otras palabras, el hecho de que los inmigrantes mexicanos puedan participar en la política de su país natal es un suceso positivo, ya que esto contribuye a la adquisición de habilidades cívico-políticas y capacidades políticas colectivas.

Sin embargo, este estudio tiene sus limitaciones. Por razones metodológicas es difícil constatar qué tan típicos son los casos aquí tratados. Las investigaciones posteriores deben incluir entrevistas con más transnacionalistas, utilizar otro tipo de técnicas cualitativas y cuantitativas, y adentrarse al tema desde un ángulo comparativo. Lo que es más, la hipótesis sobre la transferencia de recursos requiere de estudios más profundos que examinen, por ejemplo, la experiencia política de los transnacionalistas antes y después del proceso de migración y transnacionalización.

\section{APÉNDICE \\ PERFILES DE TRANSNACIONALISTAS SELECTOS}

Aragón, Julio César. Nacido en México; residente legal/ciudadano estadounidense; residente por largo tiempo, vive en Providence, Rhode Island. Presidente de la Asociación Social, Cultural y Deportiva Mexicana de Rhode Island; fundador del Political Affairs Bureau; miembro del CC-IME; afiliado a la CDPME.

Arreola, José Artemio. Nacido en México; residente legal/ciudadano estadounidense; residente por largo tiempo, vive en Chicago. Activista comunitario; líder del SEIU y conserje de escuela; líder de la Federación de Clubes Michoacanos en Illinois (FEDECMI) y el fReBIMICH; miembro del CC-IME; afiliado a la CDPME.

De la Garza, Luis. Nacido en México; residente legal/ciudadano estadounidense; residente por unos 30 años, vive en Farmers Branch, Texas; domina los idiomas español e inglés. Ingeniero y empresario; miembro de la Organización de Mexicanos en el Exterior (OEM); el National Council of Mexican American Professionals and Business Leaders (Texas); ex-presidente de la CimE-Texas; Director ejecutivo del Centro Comunitario Mexicano; Secretario de Relaciones Exteriores de la League of United Latin American Citizens (LULAC); miembro del Partido Revolucionario Institucional (PRI) en México; miembro del CC-IME, la Alianza de Inmigrantes Mexicanos en el Extranjero (antes consejeros del CC-IME), y miembro principal de la CDPME.

Dominguez, Rufino. Nacido en México; residente legal/ciudadano estadounidense; residente por más de 20 años, vive en California central; domina los idiomas español e inglés. Educación universitaria parcial; organizador comunitario; ex-trabajador migrante agrícola; Coordinador General del Frente Indígena de Organiza- 
ciones Binacionales (FIOB, antes Frente Indígena Oaxaqueño Binacional); ex-director del California Rural Legal Assistance (CRLA.); editor del boletín El Tequio; creador/ administrador del portal de Internet del FIOB; presentador del programa de televisión «Indigenous Awakening» en California central; recibió el Leadership for a Changing World Award en 2001; afiliado a la CDPME.

Gómez de Lara, José Guadalupe. Nacido en México; residente legal/ciudadano estadounidense; residente por largo tiempo, vive en Orange County, California; domina los idiomas español e inglés. Dueño de un negocio; miembro y ex-presidente de la Federación de Clubes Zacatecanos del Sur de California (FCZSC); co-fundador del FCZ; ex-presidente del COFREM; miembro del CC-IME; afiliado a la CDPME.

Gutiérrez, José Luis. Nacido en México; residente legal/ciudadano estadounidense; residente por más de 20 años, vive en Chicago; domina los idiomas español e inglés. Educación universitaria; activista comunitario; fundador del Club Morelia; presidente de la FEDECMI (2001-2005); representante del FREBIMICH; co-fundador de la NALACC; nombrado director de la Oficina de Asuntos de Inmigración del Gobernador de Illinois; miembro del CC-IME; afiliado a la CDPME.

Martínez-Saldaña, Jesús. Nacido en México; residente legal/ciudadano estadounidense; residente por largo tiempo en California/residencia binacional. Ex-profesor de Estudios Chicanos y Latinoamericanos en la California State University en Fresno; legislador estatal por el estado de Michoacán desde 2004 y presidente de la Camisón de Asuntos sobre Migrantes; miembro principal de la CDPME.

Mújica, Jorge. Nacido en México; residente legal/ciudadano estadounidense; residente por más de 20 años, vive en Chicago. Fundador de la Coalición Internacional de Mexicanos en el Extranjero (CIME) con sede en Chicago; activista sindical; miembro de la OEM; representante del Partido de la Revolución Democrática (PRD); afiliado a la CDPME.

Olamendi, Carlos. Nacido en México; residente legal/ciudadano estadounidense; residente por unos 30 años, vive en Laguna Niguel, California; domina los idiomas español e inglés. Restaurantero/Director ejecutivo de CoR International; miembro del Latin American Trade Forum; la junta del Mission Hospital; Hispanic 100 Club; Lincoln-Juarez Club; National Council of Mexican American Professionals and Business Leaders; New Coalition for Comprehensive Immigration and Security Reform, y la Coalición Internacional de Mexicanos en el Extranjero (CIME). Nombrado parte del Comité de Consejeros a la Presidencia en el Área de Artes (John F. Kennedy Center for the Performing Arts) por el ex-presidente George W. Bush y consejero económico del comité de transición del gobernador de California, Schwarzenegger; consejero del CC-IME; miembro principal de la CDPME.

Pelayo, Luis. Nacido en México; residente legal/ciudadano estadounidense; residente por casi 30 años, vive en Chicago. Educación universitaria; presidente de Pelayo and Associates; fundador/presidente del Concilio Hispano; activista comunitario; consejero del CC-IME; miembro principal de la CDPME.

110 PRIMER SEMESTRE 2009

MIGRACIÓN Y DESARROLLO 
Rodriguez-Oceguera, Primitivo. Nacido en México; ex-activista inmigrante y comunitario en Chicago; domina los idiomas español e inglés. Posgrado universitario; historiador y analista político; ex-consejero del Subsecretario de Derechos Humanos en la Secretaría de Relaciones Exteriores (SER) de México; coordinador de la CDPme en la Ciudad de México.

Ross-Pineda, Raúl. Nacido en México; residente legal/ciudadano estadounidense; residente por unos 30 años, vive en Chicago; domina los idiomas español e inglés. Analista político; escritor; director fundador de la revista MX Sin Fronteras; ex-director de la Agenda Mexicana para el American Friends Service Committee; miembro del CC-IME; miembro principal y co-fundador de la CDPME.

Zaragoza, Florencio I. Nacido en México; residente legal/ciudadano estadounidense; residente por largo tiempo, vive en Tucson, Arizona; domina los idiomas español e inglés. Educación universitaria; presidente de la Fundación México y MX; miembro principal de la CDPME.

\section{BIBLIOGRAFÍA}

BADA, Xochitl, Jonathan Fox y Andrew Seele (2006), Invisible No More: Mexican Migrant Civic Participation in the United States, Washington, DC, Mexico Institute, Woodrow Wilson International Center for Scholars.

Brady, Henry E., Sidney Verba y Kay Lehman Schlozman (1995), «Beyond ses: A Resource Model of Political Participation", American Political Science Review, 89: 271-290.

Cano, Gustavo y Alexandra Délano (2004), «The Institute of Mexicans Abroad: The Day

After... After 156 Years», artículo presentado en el Annual Meeting of the American Political Science Association, Chicago, 2-5 de septiembre.

(2002), The Chicago-Houston Report: Political Mobilization of Mexican Immigrants in American Cities, San Diego, Center for U.S.-Mexican Studies, University of California. http://repositories.cdlib.org/usmex/cano/

CANTo, Minerva (2002), «Delegation of Influential Immigrants is Lobbying Mexican Leaders for the Right to Vote from Abroad», Orange County Register, 13 de marzo, 1.

y Dena Bunis (2003), «Latino Activists Resume Push for Migration Accord», Orange County Register, 1 de mayo.

De la Garza, Rodolfo O. (2004), «Latino Politics», Annual Review of Political Science, 7: 91-123. y Myriam Hazan (2003), Looking Backward Moving Forward: Mexican Organizations

in the U.S. as Agents of Incorporation and Dissociation, Claremont, The Tomás Rivera Policy Institute.

DeSirio, Louis et al. (2003), Immigrant Politics at Home and Abroad: How Latino Immigrants

Engage the Politics of their Home Communities and the United Status, Claremont, Tomas Rivera Policy Institute.

Elias, Tom (1998), «Dual Nationality May Reduce Latino Clout», Daily Breeze, 8 de junio. Escobar, Cristina (2004), «Dual Citizenship and Political Participation: Migrants in the Interplay of United States and Colombian Politics», Latino Studies, 2: 45-69. 
Fitzgerald, David (2000), Negotiating Extra-Territorial Citizenship: Mexican Migration and the Transnational Politics of Community, ccis, Monograph 2, La Jolla, University of California, San Diego.

Foner, Nancy (2000), «Transnational Ties», From Ellis Island to JFK: New York's Two Great Waves of Immigration, New Haven, Yale University Press.

Glick Schiller, Nina (1999), «Transmigrants and Nation-States: Something Old and Something New in the U.S. Immigrant Experience», en C. Hirschman et al. (editores), The Handbook of International Migration, New York, Russell Sage Foundation, 94-119.

GoldRING, Luin (2002), "The Mexican State and Transmigrant Organizations: Negotiating the Boundaries of Membership and Participation», Latin American Research Review, 37. 3: 55-99.

GonZÁlez GutiérRez, Carlos (1995), «La organización de los inmigrantes mexicanos en Los Ángeles: la lealtad de los oriundos», Revista Mexicana de Politica Exterior, (primavera) 46: 59-101.

Guarnizo, Luis Eduardo, Alejandro Portes, y William Haller (2003), «From Assimilation to Transnationalism: Determinants of Transnational Political Action among Contemporary Migrants», American Journal of Sociology, 108: 1211-1248.

Hritzuk, Natasha, y David K. Park (2000) "The Question of Latino Participation: from an SES to a Social Structural Explanation", Social Science Quarterly, 81.1 (marzo): 151-167.

Huntington, Samuel (2004), Who Are We? The Challenges to America's National Identity, New York, Simon and Schuster.

Jones-Correa, Michael (2001), «Under Two Flags: Dual Nationality in Latin America and Its Consequences for Naturalization in the United States», International Migration Review, 35: 997-1029.

Landolt, Patricia, Lilian Autler y Sonia Baires (2003), «Del hermano lejano al hermano mayor: La dialéctica del transnacionalismo salvadoreño», en Alejandro Portes et al. (editores), La globalización desde abajo: transnacionalismo inmigrante y desarrollo, la experiencia de Estados Unidos y América Latina, México, Facultad Latinoamericana de Ciencias Sociales Sede-México, 123-158.

La Opinión, Anuncio, 1 de julio, 2006, 8B.

Martínez-Saldaña, Jesús (2002), «Segunda Parte. Participación política migrante: Praxis cotidiana de ciudadanos excluidos», en Leticia Calderón-Chelius y Jesús MartínezSaldaña (editores), La dimensión política de la migración mexicana, México, Instituto Mora, 159-329.

(2003), «Reclamando el poder: los ciudadanos en el exterior y su impacto en la política mexicana», Migraciones Internacionales, 2.2 (julio-diciembre), 96-120.

y Raúl Ross-Pineda (2002), «Suffrage for Mexicans Residing Abroad», en David Brooks y Jonathan Fox (editores), Cross-Border Dialogues: U.S. Mexico Social Movement Networking, La Jolla: Center for U.S.-Mexican Studies, University of California, San Diego, 275-292.

McCann, James A., Wayne Cornelius y David Leal (2007), «Engagement in Campaigns and Elections South of the Border Pull Mexican Immigrants Away from U.S. Politics? Evidence from the 2006 Mexican Expatriate Study», artículo presentado en el Latin American Studies Association Annual Meeting, Montreal, 5-8 de septiembre.

112 PRIMER SEMESTRE 2009 MIGRACIÓN Y DESARROLLO 
Moctezuma-Longoria, Miguel (2003), "Territorialidad socio-cultural y política de los clubes zacatecanos en Estados Unidos», Red Internacional de Migración y Desarrollo, 1 (octubre), 1-25.

Portes, Alejandro, Cristina Escobar, y Alexandra Walton Radford (2007), «Immigrant Transnational Organizations and Development: A Comparative Study», International Migration Review, 48, 1 (primavera), 242-82.

(2003), «Conclusión: Hacia un nuevo mundo. Los Orígenes y efectos de las actividades transnacionales», en Alejandro Portes et al. (editores), La globalización desde abajo: transnacionalismo inmigrante y desarrollo, la experiencia de Estados Unidos y América Latina, México, Facultad Latinoamericana de Ciencias Sociales Sede-México, $377-$ 395.

Pickus, Noah M. (1997), Becoming American/American Becoming. Final Report of the Duke University Workshop on Immigration and Citizenship. http://www.pubpol.duke.edu/ people/faculty/pickus/immigration.

Renshon, Stanley A. (2005), The 50\% American: Immigration and National Identity in an Age of Terror, Washington, D.C., Georgetown University Press.

Rivera-Salgado, Gaspar (2006), "Mexican Migrants and the Mexican Political System», en Xochitl Bada et al. (editores), Invisible No More: Mexican Migrant Civic Participation in the United States, Washington, D.C., Mexico Institute, Woodrow Wilson International Center for Scholars, 31-34.

(2002), "Cross-Border Grassroots Organizations and the Indigenous Migrant Experience», en David Brooks y Jonathan Fox (editores), Cross-Border Dialogues: U. S.-Mexico Social Movement Networking, La Jolla, Center for U.S.-Mexican Studies, University of California, San Diego, 259-276.

(1999), «Mixtec Activism in Oaxacalifornia: Transborder Grassroots Political Strategies", American Behavioral Scientist, 42: 1439-1458.

Rodríguez-Oceguera, Primitivo (2005), "Así se ganó el voto», MX sin Fronteras (agosto): 14-22. Ross, Raúl y Nayamín Martínez-Cossío (2004), "Cronología», en Gonzalo Badillo (editor), La puerta que llama: los derechos políticos de los mexicanos en el extranjero, México, Senado Mexicano.

Schmidt, Steffen et al. (2008), American Government and Politics Today: 2007-2008. Texas Edition, Belmont, Thomson/Wadsworth.

SHAIn, Yossi y Aharon Barth (2003), "Diasporas and International Relations Theory», International Organization, 57: 449-479.

SMITH, Robert (1998), «Transnational Localities: Community, Technology and the Politics of Membership within the Context of México and U.S. Migration», en Michael Peter Smith y Luis Eduardo Guarnizo (editores), Transnationalism from Below, Comparative Urban \& Community Research 6. New Brunswick: Transaction Publishers, 196-238. Smith, Michael Peter, y Luis Eduardo Guarnizo (1998), Transnationalism from Below, News

Brunswick, New Jersey, Transaction Publishers.

TRUAX, Eileen (2006a) «Cierra campaña para voto postal», La Opinión, 16 de enero. (2006b), «Federaciones contra los planes antiinmigrantes», La Opinión, 16 de febrero.

Verba, Sidney, Kay Lehman Schlozman y Henry E. Brady (1995), Voice and Equality: Civic Voluntarism in American Politics, Cambridge, Harvard University. 
ZABIN, Carol y Luis Escala-Rabadán (1998), «Mexican Hometown Associations and Mexican Immigrant Political Empowerment in Los Angeles», Nonprofit Sector Research Fund Working Paper Series, The Aspen Institute. 